\title{
Increased plasma BMP-2 levels are associated with atherosclerosis burden and coronary calcification in type 2 diabetic patients
}

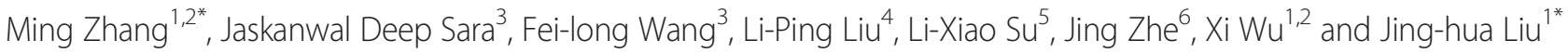

\begin{abstract}
Background: Although Bone morphogenetic protein-2 (BMP-2) is a known mediator of bone regeneration and vascular calcification, to date no study has investigated the relationship between BMP-2 and type 2 diabetes mellitus (T2DM) and its possible role in coronary artery disease (CAD). The purpose of this study is to evaluate the relationship of BMP-2 with atherosclerosis and calcification in patients with T2DM.

Methods: 124 subjects were enrolled in this study: 29 patients with T2DM and CAD; 26 patients with T2DM and without CAD; 36 patients with CAD and without T2DMand 34 without T2DM or CAD (control group). Severity of coronary lesions was assessed using coronary angiography and intravascular ultrasound (IVUS). Plasma BMP-2 levels were quantified using a commercially available ELISA kit.

Results: Compared to the control group, the mean plasma BMP-2 level was significantly higher in T2DM patients with or without CAD (20.1 \pm 1.7 or $19.3 \pm 1.5 \mathrm{pg} / \mathrm{ml}$, vs $17.2 \pm 3.3 \mathrm{pg} / \mathrm{ml}, \mathrm{P}<0.001)$. In a multivariable linear regression analysis, both T2DM and CAD were significantly and positively associated with BMP-2 (Estimate, 0.249; standard error (SE), $0.063 ; p<0.0001$; Estimate, $0.400 ; \mathrm{SE}, 0.06 ; p<0.0001)$. Plasma BMP-2 was also strongly correlated with glycosylated hemoglobin A1c (HbA1c) (Spearman $\rho=-0.31 ; p=0.0005)$. SYNTAX score was also significantly associated with BMP-2 (Spearman $\rho=0.46 ; p=0.0002$ ). Using the results from IVUS, plasma BMP-2 levels were shown to positively correlate with plaque burden (Spearman $\rho=0.38, P=0.002$ ) and plaque calcification (Spearman $\rho=0.44, P=0.0003$ ) and to negatively correlate with lumen volume (Spearman $\rho=0.31, P=0.01$ ).
\end{abstract}

Conclusions: Our study demonstrates that patients with T2DM had higher circulating levels of BMP-2 than normal controls. Plasma BMP-2 levels correlated positively with plaque burden and calcification in patients with T2DM.

Keywords: Bone morphogenetic protein-2, Type 2 diabetes mellitus (T2DM), Coronary artery disease, Plaque burden and plaque calcification

\section{Introduction}

Bone morphogenetic protein-2 (BMP-2), a member of the transforming growth factor (TGF) superfamily, plays a major role in the regulation of conventional and ectopic osteogenesis [1,2]. A growing body of evidence indicates that BMP-2 signaling plays an important role in vascular disease, including atherosclerosis, plaque instability $[3,4]$, and vascular calcification and inflammation $[5,6]$.

\footnotetext{
* Correspondence: zhangming2279@hotmail.com; liujinghua@vip.sina.com 'Department of Cardiology, Beijing Anzhen Hospital, Capital Medical University, Beijing 100029, PR China

Full list of author information is available at the end of the article
}

Specifically, increased levels of BMP-2 exert proinflammatory, proatherogenic effects by inducing oxidative stress and endothelial dysfunction and have been shown to promote plaque calcification by inducing an osteogenic phenotype in vascular smooth muscle cells (VSMCs) $[7,8]$ In the coronary arteries, BMP-2 is regulated by inflammatory stimuli [9] and its upregulation in the vasculature may also be related to oxidative stress, hyperglycemia and hyperlipidemia [10,11].

Diabetes mellitus substantially increases the risk of cardiovascular and peripheral arterial disease [12] and is associated with an increased risk of cardiovascular mortality. People with type 2 diabetes (T2DM) have approximately a 
doubled risk of cardiovascular disease, compared with people without diabetes, even after adjusting for established cardiovascular risk factors [13]. The pathophysiology of vascular disease in diabetes involves abnormalities in endothelial and VSMCs, and alterations in platelet function $[14,15]$. Vascular calcification is also more common in patients with diabetes and is associated with increased mortality, stroke and amputations [16-19]. Highly prevalent vascular calcification in patients with T2DM may be related to biochemical changes [20,21]. For example, Chen et al. found that high serum glucose levels were associated with an increased expression of Cbfa1 and BMP-2 and enhanced the calcification of VSMCs [22,23].

Neverthesless, the role of high glucose concentrations in atherosclerosis is complex and requires clarification. In addition, while BMP-2 is a known mediator of vascular calcification [19], the role of BMP-2 in the development of atherosclerosis remains uncertain, particularly amongst T2DM patients. In this study, we measured plasma BMP-2 levels in patients with CAD and T2DM and examined the association between BMP-2 and clinicopathological parameters of coronary atherosclerotic disease.

\section{Methods}

\section{Study subjects}

Between 6/2/2011 and 6/17/2013, a total of 124 consecutive patients undergoing diagnostic coronary angiography for the evaluation of coronary artery disease (CAD) were recruited. The diagnosis of CAD was made using angiography and was defined as angiographic evidence of $\geq 50 \%$ luminal narrowing in at least one segment of a main epicardial coronary artery. Study subjects were divided into four groups: (i) patients without CAD or T2DM $(n=34)$; (ii) patients with T2DM only $(\mathrm{n}=26)$; (iii) patients with CAD only ( $\mathrm{n}=31$ ) and (iv) patients with T2DM and CAD ( $\mathrm{n}=29$ ). Diabetes mellitus was diagnosed based upon the WHO guidelines or current intake of hypoglycemic agents [24].

Patients with a history of significant concomitant disease including hepatic failure, renal failure, hepatitis, cardiomyopathy, congenital heart disease, bleeding disorders, previous thoracic irradiation therapy, and malignant diseases were excluded from the study. This study was approved by the Beijing Anzhen Hospital Ethics Committee of Capital Medical University, and informed consent was obtained from all participants.

\section{Plasma collection and storage}

Blood samples were collected in EDTA-containing tubes (BD, Franklin Lakes, NJ, USA) and plasma was isolated within 1 hour by centrifugation at $1900 \times \mathrm{g}$ for 10 minutes at $4^{\circ} \mathrm{C}$ to remove blood cells, and then at $16,000 \times \mathrm{g}$ for 10 minutes at $4^{\circ} \mathrm{C}$ to remove additional cellular nucleic acid attached to cell debris. Plasma samples were stored at $-80^{\circ} \mathrm{C}$ prior to being analyzed.

\section{Specific BMP-2 ELISA}

BMP-2 levels were quantified using a commercially available ELISA kit (Quantikine, BMP-2 Immunoassay, R\&D Systems, MN USA). All samples were assayed according to the manufacturer's instructions and were tested in duplicate by personnel blinded to each patient's group. The optical density of each well was determined using a microplate reader at $450 \mathrm{~nm}$. No interference and no cross-reactivity were expected based on the manufacturer's instructions.

\section{Coronary angiography and assessment of CAD severity}

Coronary angiography was performed according to standard methods. Each coronary angiogram was scored by two independent investigators according to the following scores: (1) Vessel score: The number of vessels with significant ( $\geq 50 \%$ ) stenosis [25]; (2) Synergy between PCI with Taxus and Cardiac surgery score (SYNTAX score), an angiographic score that determines the severity and complexity of disease in coronary lesions of $\geq 50 \%$ stenosis, in vessels $\geq 1.5 \mathrm{~mm}$ [26-28]. All angiographic variables of the SYNTAX score were computed by two experienced cardiologists who were blinded to procedural and clinical data. In cases of disagreement, the final decision was reached by consensus.

Intravascular ultrasound examination and image analysis Virtual histology-Intravascular Ultrasound (IVUS) examinations were performed using a $20 \mathrm{MHz}, 2.9 \mathrm{~F}$ phasedarray IVUS catheter (Eagle Eye Gold, Volcano Corporation, Rancho Cordova, CA, USA). Vessel and lumen borders were manually contoured for all frames in each coronary segment. Quantitative IVUS measurements inclued vessel volume, lumen volume, plaque volume and percent plaque burden. Radiofrequency IVUS derived plaque components were color-coded and reported as the absolute plaque volume of VH-IVUS parameters [fibrous (dark green), fibrofatty (light green), necrotic core (red) and dense calcium (white)] to assess for plaque composition [29,30]. Simpson's rule for volumetric measurements was used. To compensate for the different segment lengths of each analyzed artery, all volumetric data were divided by segment length and displayed as volume index $(\mathrm{mm} 3 / \mathrm{mm})$ [31].

\section{Statistical analysis}

Normality of distribution was assessed using KolmogorovSmirnov test. Comparisons between 2 groups were performed using Fisher's test, Student's $t$ test or Mann-Whitney $U$ test. For comparisons between more than 2 groups, one-way ANOVA and Tukey-Kramer HSD (honestly significant difference) tests were used as appropriate. Pearson 
$\chi^{2}$ test and Spearman $\rho$ test were used to compare qualitative and quantitative variables as appropriate. Correlations between BMP-2 and other variables were calculated using Pearson's correlation coefficient for symmetrically distributed data and Spearman's correlation coefficient for skewed variables. Univariate and multivariable linear regression analyses were performed to identify variables that were associated with BMP-2 after adjusting for potential confounders. All tests were two-sided and a $p<0.05$ was considered statistically significant. All statistical analyses were performed using JMP 10 software (SAS Institute, Inc., Cary, NC, USA).

\section{Results}

Baseline clinical characteristics of study participants

Among the 124 subjects, mean age was $59.4 \pm 11.7$ years and the proportion of males was $66 \%$. Mean BMP-2 in the study population was $18.8 \pm 2.5(\mathrm{pg} / \mathrm{ml})$. Of the $124 \mathrm{pa}-$ tients, 60 were diagnosed as having CAD and 45 patients had T2DM, and the remainder had neither disease $(n=34)$.
Prevalence of hypertension, dyslipidemia and smoking was 83 (69\%), 86 (71\%) and 51 (42\%) respectively.

\section{Elevated plasma BMP-2 levels are associated with the presence of T2DM}

BMP-2 levels differed significantly across all groups (Table 1). Compared to subjects without T2DM or CAD, mean plasma BMP-2 levels were significantly higher in patients with T2DM with or without CAD $(20.1 \pm 1.7$ or $19.3 \pm 1.5 \mathrm{pg} / \mathrm{ml}$ vs $17.1 \pm 3.3, \mathrm{P}<0.001$, respectively) (Table 1, Figure 1).

Table 2 demonstrates the results of an univariate linear regression analyses to determine the association between clinical variables and BMP-2. Random blood glucose levels, and the presence of T2DM and CAD were significantly associated with BMP-2. High-sensitivity C-reactive protein showed a tendency towards a positive association with BMP-2 with borderline statistical significance. After adjustment for traditional cardiovascular risk factors, random blood glucose, HbA1c, T2DM and CAD were

Table 1 Baseline patient characteristics amongst all patients

\begin{tabular}{|c|c|c|c|c|c|}
\hline Clinical variables & Normal $(n=34)$ & T2DM $(n=26)$ & CAD $(n=31)$ & T2DM + CAD $(n=29)$ & $P$ value \\
\hline Age, yr & $58.0 \pm 11$ & $60 \pm 2.3$ & $57 \pm 2.2$ & $61.6 \pm 2.0$ & 0.41 \\
\hline Male Gender, n (\%) & $21,(61)$ & $20,(77)$ & $21,(68)$ & $20,(61)$ & 0.50 \\
\hline Body mass index, $\mathrm{kg} / \mathrm{m} 2$ & $26 \pm 2.9$ & $27 \pm 3.7$ & $27 \pm 4.0$ & $30 \pm 3.7$ & 0.07 \\
\hline Hypertension, n (\%) & $18(56)$ & $21(84)$ & $119(61)$ & $25(76))$ & 0.08 \\
\hline Dyslipidemia, n (\%) & $8(25)$ & $5(21)$ & $19(61)$ & $25(76)$ & 0.63 \\
\hline Smoking, n (\%) & 15(76) & $12(48)$ & $15(48)$ & $9(27)$ & 0.23 \\
\hline Blood glucose, mmol/L & $5.6 \pm 1.1$ & $6.2 \pm 2.2$ & $5.5 \pm 1.0$ & $7.6 \pm 2.8$ & 0.004 \\
\hline Triglycerides, $\mathrm{mmol} / \mathrm{L}$ & $1.7 \pm 1.1$ & $2.0 \pm 0.3$ & $2.0 \pm 0.2$ & $1.9 \pm 0.2$ & 0.63 \\
\hline Total cholesterol, mmol/L & $4.4 \pm 0.2$ & $4.3 \pm 0.2$ & $4.4 \pm 0.9$ & $4.2 \pm 0.2$ & 0.92 \\
\hline Low-density lipoprotein, mmol/L & $2.7 \pm 0.9$ & $2.7 \pm 0.8$ & $2.7 \pm 0.3$ & $2.5 \pm 0.9$ & 0.62 \\
\hline High-density lipoprotein, mmol/L & $1.0 \pm 0.04$ & $1.0 \pm 0.04$ & $1.0 \pm 0.03$ & $0.98 \pm 0.04$ & 0.80 \\
\hline Hs CRP, mg/L, Median (Q1, Q3) & $1.5(0.7,3.7)$ & $0.64(0.4,1.6)$ & $1.2(0.7,2.9)$ & $1.4(0.7,6.5)$ & $<0.001$ \\
\hline Cr, $\mu$ mol/L Median (Q1, Q3) & $72(65,85)$ & $76(67,86)$ & $76(66,88)$ & $77(63,84)$ & 0.9 \\
\hline Ejection fraction, \% & $64 \pm 5.7$ & $63 \pm 6.7$ & $64 \pm 7.3$ & $62.2 \pm 6.2$ & 0.8 \\
\hline LVEDD, mm & $47 \pm 4.9$ & $50.1 \pm 4.5$ & $48 \pm 5.3$ & $47 \pm 4.6$ & 0.20 \\
\hline BMP-2 (pg/mL) & $17.1 \pm 0.6$ & $19.2 \pm 0.3$ & $18.7 \pm 0.3$ & $20.1 \pm 0.3$ & $P<0.001$ \\
\hline $\mathrm{HbA1c}(\mathrm{n} \%)$ & $5.9 \pm 1.52$ & $7.3 \pm 1.6$ & $5.8 \pm 0.7$ & $8.23 \pm 1.6$ & $P<0.001$ \\
\hline \multicolumn{6}{|l|}{ Medications on admission } \\
\hline Aspirin, No. (\%) & $24(71)$ & 18(69) & $24(77)$ & $26(78)$ & 0.78 \\
\hline Clopidogrel, No. (\%) & 13(38) & $12(46)$ & $16(52)$ & $20(61)$ & 0.32 \\
\hline Beta-blockers, No. (\%) & $14(41)$ & $12(48)$ & $14(45)$ & $16(48)$ & 0.93 \\
\hline ACEI/ARB, No. (\%) & $14(42)$ & $17(71)$ & $16(76)$ & 23(85) & 0.0017 \\
\hline Statins, No. (\%) & $8(23.5)$ & $15(58)$ & $15(48)$ & $22(67)$ & 0.0025 \\
\hline Insulin, No. (\%) & $0(0)$ & $17(65)$ & $0(0)$ & $23(79)$ & $<0.001$ \\
\hline Oral hypoglycemics, No. (\%) & $0(0)$ & $18(69)$ & $0(0)$ & 25(86) & $<0.001$ \\
\hline
\end{tabular}

BMP-2, Bone Morphogenic Protein-2; CAD, Coronary artery disease; HbA1c; Glycosylated hemoglobin A1c; hsCRP, High sensitivity C-reactive protein; LVEDD, Left ventricular end diastolic diameter; $\mathrm{Cr}$, Serum creatinine; T2DM, Type II Diabetes Mellitus. 


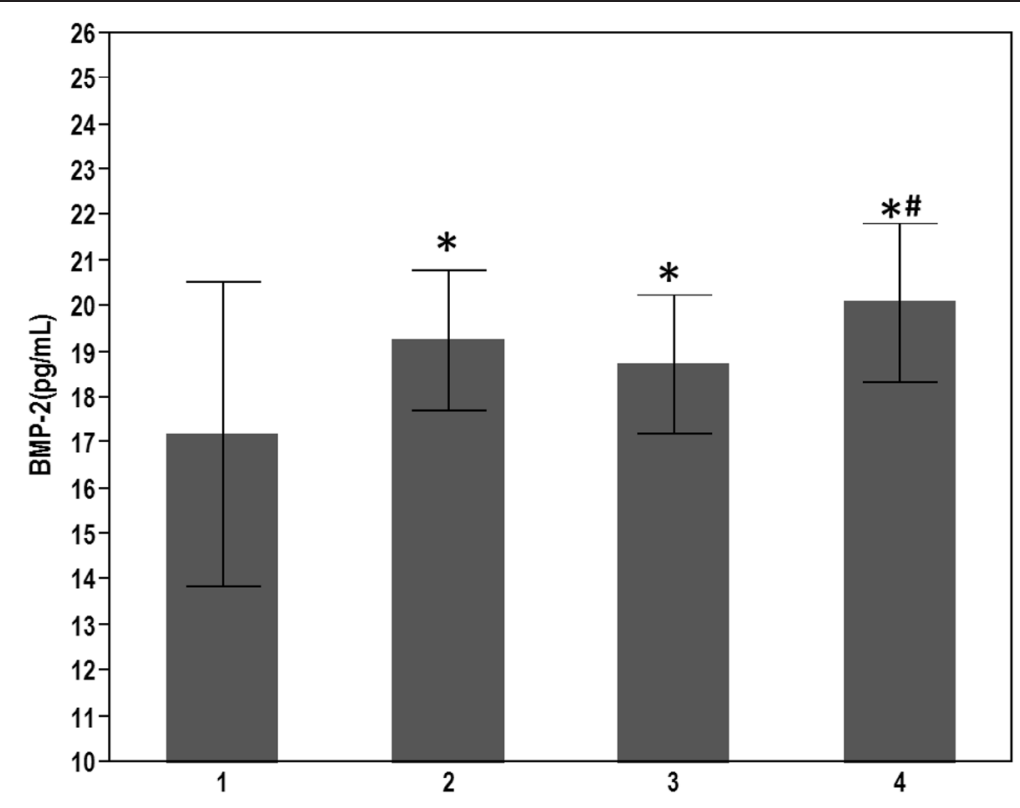

Figure 1 Elevated plasma BMP-2 levels are associated with the presence of DM and CAD. Bars represent average plasma BMP-2 levels amongst patients in each group. T-bars indicate standard deviation. Groups are as follows: 1; patients without CAD or T2DM, 2; patients with T2DM only; 3, patients with CAD only and 4, patients with T2DM and CAD. ( ${ }^{*} p<0.05$ as compared to group without CAD and T2DM; \# $p<0.05$ as compared to group with CAD only).

significantly associated with BMP-2 levels (Estimate, 0.32; standard error, 0.16; $p=0.04$; Estimate, 0.30; standard error, 0.13; $p=0.021$; Estimate, 1.565; standard error, 0.434; $p=0.0005$; Estimate, 1.299; standard error, 0.425; $p=0.0028$, respectively) (Table 3 ). An interaction term between CAD and T2DM was included in our multivariable analysis and proved not to be significant $(\mathrm{p}=0.46)$ and so was excluded from our final model. Plasma BMP-2

Table 2 Association between BMP-2 with type II diabetes mellitus and coronary artery disease after adjusting for traditional cardiovascular risk factors

\begin{tabular}{llll}
\hline Variable & Estimate & Standard error & $\mathbf{P}$ \\
\hline Age (Increase by 1 year) & 0.57 & 0.42 & 0.19 \\
Male gender & -0.032 & 0.07 & 0.68 \\
Body mass index & -0.01 & 0.009 & 0.30 \\
Hypertension & -0.11 & 0.08 & 0.18 \\
Dyslipidemia & -0.006 & 0.08 & 0.94 \\
Smoking & -0.051 & 0.08 & 0.50 \\
Random blood glucose, mmol/L & 0.12 & 0.05 & 0.027 \\
Increase by 1 mmol/L) & & & 0.08 \\
Hs-CRP, mg/L (Increase by 1 mg/L) & 0.33 & 0.19 & 0.0079 \\
HbA1c (n \%) (Increase by 1\%) & 0.17 & 0.06 & 0.0002 \\
T2DM & -0.39 & 0.11 & 0.004 \\
CAD & -0.26 & 0.09 & \\
\hline
\end{tabular}

Data are expressed as parameter estimates with standard errors. CAD, Coronary artery disease; $\mathrm{HbA1C}$, glycosylated hemoglobin A1C; $\mathrm{Hs}-\mathrm{CRP}$, Highly sensitive C-reactive protein; T2DM, Type II diabetes mellitus. levels correlated positively with HbA1c (Spearman $\rho=0.31$; $p=0.0005$ ) (Figure 2).

\section{Assessment of CAD severity using coronary angiography} CAD with three-vessel disease was more prevalent in the CAD and T2DM group compared with the CAD only group $(48.9 \%$ vs $20.6 \%, p=0.002)$. When assessing the severity of CAD using the SYNTAX score, the mean SYNTAX score in CAD patients with T2DM were significantly higher than in patients with CAD only $(29.3+$ 6.3 vs. $11.7+6.2, p<0.001)$. Plasma levels of BMP-2 correlated positively with SYNTAX score (Spearman $\rho=0.46$; $p=0.0002$ ) (Figure 3). In a multivariable linear regression analysis, SYNTAX score was significantly associated with BMP-2 levels (Estimate, 2.5, standard error, 0.79; $p=0.0026$ ) (Table 4).

Table 3 Association between BMP-2 levels and plaque volume and dense calcium in patients with coronary artery disease

\begin{tabular}{llll}
\hline Variable & \multicolumn{3}{l}{ Adjusted for traditional risk } \\
\cline { 2 - 4 } & Estimate & SE & $\mathbf{P}$ \\
\hline T2DM & 1.565 & 0.434 & 0.0005 \\
CAD & 1.299 & 0.425 & 0.0028 \\
Random blood glucose, & 0.32 & 0.160 & 0.04 \\
mmol/l (Increase by 1 mmol/l) & & & \\
HbA1c, \% & 0.30 & 0.13 & 0.021 \\
\hline
\end{tabular}

CAD, Coronary artery disease; HbA1C, glycosylated hemoglobin; T2DM, Type II diabetes mellitus. 


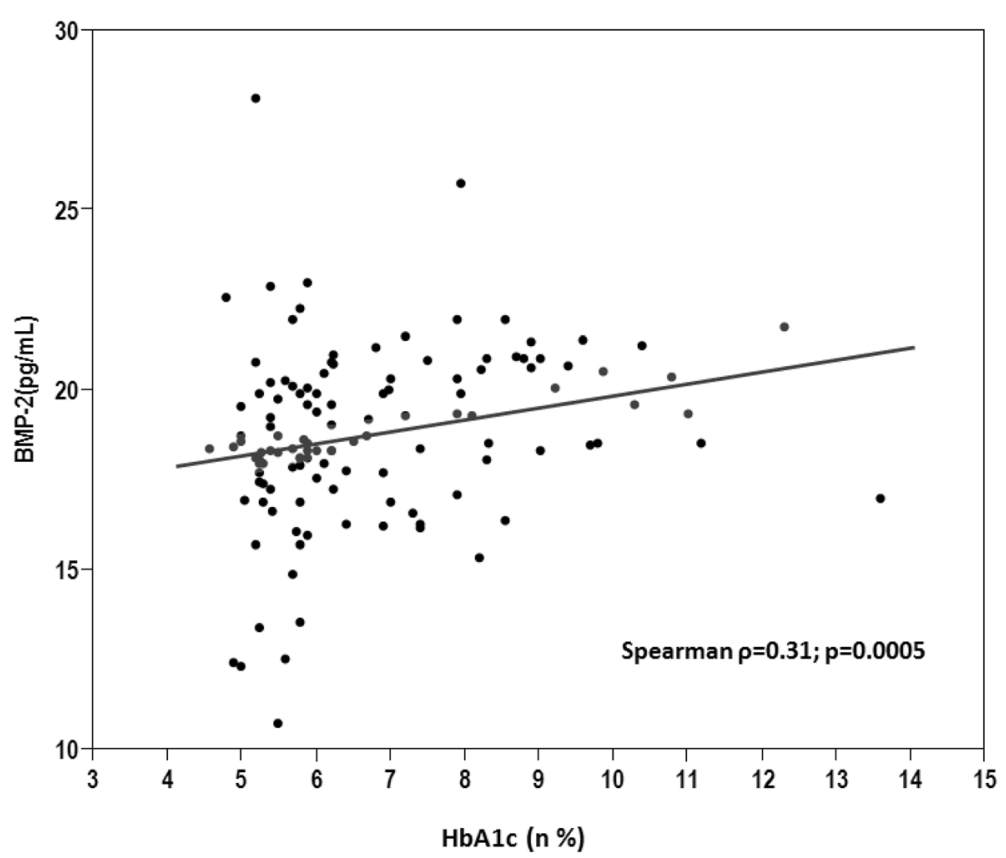

Figure 2 Scatter diagram demonstrating the correlation between BMP-2 levels and $\mathrm{HbA} 1 \mathrm{c}$. HbA1c is significantly positively correlated with plasma BMP-2 levels.

Assessment of plaque burden and calcium density using intravascular ultrasound

Data derived from IVUS are shown in Table 5. Plaque volume index and dense calcium were significantly higher in patients with CAD and T2DM compared to patients with CAD only $(13.9[12,15]$ vs. $9.3[8.2,12]$; $p<0.0001 ; 2.7[2.1,3.8]$ vs. $2.0[1.3,3.0] ; p=0.023)$. Lumen volume index in the CAD and T2DM group was significantly lower compared with that in the CAD group (4.4 $[3.8,6.5]$ vs. $5.8[4.8,6.5] ; p<0.001$ ) (Table 5).

In a multivariable linear regression analysis, lumen volume, plaque volume index and plaque dense calcium

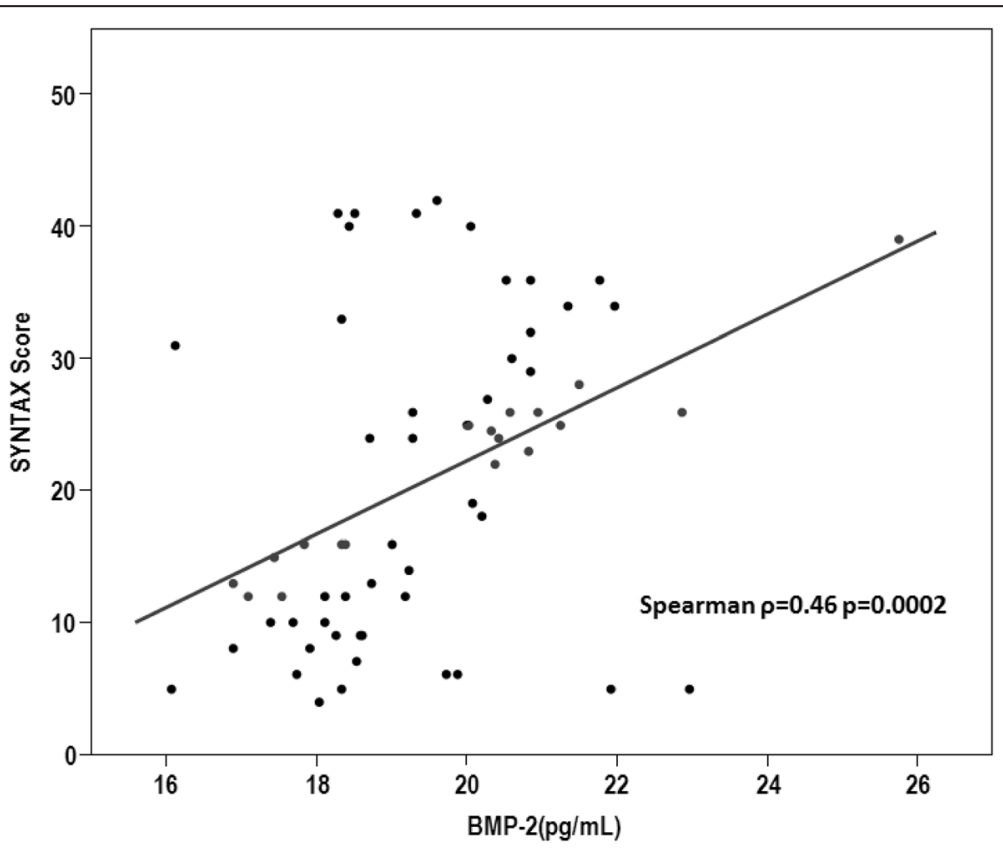

Figure 3 Scatter diagram demonstrating the correlation between BMP-2 levels and SYNTAX score. SYNTAX is significantly positively correlated with plasma BMP-2 levels. 
Table 4 Virtual Histology-Intravascular Ultrasound parameters amongst study groups

\begin{tabular}{|c|c|c|c|c|c|c|}
\hline \multirow[t]{2}{*}{ Variable } & \multicolumn{3}{|l|}{ Univariate } & \multicolumn{3}{|c|}{ Adjusted for risk factors } \\
\hline & Estimate & SE & $\mathbf{P}$ & Estimate & SE & $\mathbf{P}$ \\
\hline SYNTAX score & 2.78 & 0.76 & 0.0005 & 2.5 & 0.79 & 0.0026 \\
\hline Lumen volume index, (mm3/mm) & -0.34 & 0.13 & 0.0126 & -0.273 & 0.13 & 0.042 \\
\hline Plaque volume index, (mm3/mm) & 0.61 & 0.25 & 0.018 & 0.58 & 0.27 & 0.035 \\
\hline Dense calcium, (mm3/mm) & 0.29 & 0.09 & 0.0028 & 0.31 & 0.10 & 0.0025 \\
\hline
\end{tabular}

CAD, Coronary artery disease; SE, Standard error; Data are expressed as parameter estimates with standard errors. Risk factors adjusted for in multivariate analysis: age, gender, hypertension, hyperlipidemia and smoking.

was significantly associated with BMP-2 (Estimate, -0.273 ; standard error, $0.13 ; p=0.042$ and Estimate, 0.58 ; standard error, $0.27 ; p=0.035$; Estimate, 0.31 ; standard error, 0.10 ; $p=0.0025$, respectively) (Table 4 ).

Plasma BMP-2 levels correlated negatively with lumen volume index (Spearman $\rho=-0.31, P=0.01$ ) (Figure 4A), and correlated positively with plaque volume index (Figure 4B), and plaque calcium density (Spearman $\rho=0.38$, $P=0.002$; Spearman $\rho=0.44, P=0.0003$, respectively) (Figure 5A). Dense calcium correlated positively with plasma HbA1c levels. (Spearman $\rho=0.29, p=0.02$ ) (Figure 5B).

\section{Discussion}

In the current study, we examined the association between plasma BMP-2 levels and CAD in patients with and without T2DM. Compared to normal subjects, plasma BMP-2 levels were significantly higher in T2DM patients with and without CAD. Plasma BMP-2 levels also correlated positively with random blood glucose levels and HbA1c. In addition, plasma BMP-2 levels were significantly higher in patients with CAD compared to those without CAD and were positively associated with the severity and extent of coronary lesions. Our IVUS findings also showed that plasma BMP-2 levels correlated positively with plaque calcification and plaque burden, which suggests an important role of BMP-2 in accelerating atherosclerotic progression and plaque calcification.

\section{BMP-2 is associated with CAD in patients with T2DM}

Recent studies have demonstrated that BMP-2 plays an important role in both physiological and pathological vascular processes [32,33]. VSMCs represent a significant source of BMP-2, which has been reported to be highly expressed in human vessels $[34,35]$. Moreover, several studies have consistently shown that BMP-2 enhances VSMC migration [36,37]. Hyperglycemia plays a key role in the regulation of the vascular inflammatory response by activating the recruitment of inflammatory cells to injured arteries. Hyperglycemia also stimulates atherosclerosis through various processes including endothelial dysfunction and cellular proliferation as well as inflammation $[22,38,39]$. Although the role of high serum glucose levels in atherosclerosis is well documented, its role in vascular calcification is more complex. Our previous study showed that high glucose levels increased BMP-2 expression, concomitantly with NF-kB activation [23]. In the current study, we extend these findings by showing that plasma BMP-2 levels correlate positively with HbA1c, suggesting that chronically elevated glucose levels may enhance BMP-2 expression in patients with T2DM.

Table 5 Univariate linear regression analysis for plasma levels of BMP-2

\begin{tabular}{|c|c|c|c|}
\hline IVUS parameter & CAD $(n=31)$ & T2DM + CAD $(n=29)$ & $P$ value \\
\hline IVUS target vessel & & & 0.51 \\
\hline$\angle A D$ & $17(55)$ & $17(51)$ & \\
\hline RCA & $9(29)$ & $7(21)$ & \\
\hline LCX & $5(16)$ & $9(27)$ & \\
\hline IVUS vessel length(mm) Median (Q1, Q3) & $24(17,35)$ & $21(14,28)$ & 0.22 \\
\hline Lumen volume index, (mm3/mm) Median (Q1, Q3) & $5.8(4.8,7.4)$ & $4.4(3.8,6.5)$ & 0.0051 \\
\hline Plaque volume index, (mm3/mm) Median (Q1, Q3) & $9.3(8.2,12)$ & $13.9(12,15)$ & $<0.001$ \\
\hline Dense calcium, (mm3/mm), Median (Q1, Q3) & $2(1.3,3)$ & $2.7(2.1,3.8)$ & 0.023 \\
\hline Fibrotic tissue, (mm3/mm), Median (Q1, Q3) & $2.8(1.8,4)$ & $3.9(2.7,5.9)$ & 0.82 \\
\hline Necrotic core, (mm3/mm), Median (Q1, Q3) & $2.3(1.5,3.5)$ & $2.8(2.2,4)$ & 0.06 \\
\hline Fibro-fatty, (mm3/mm) , Median (Q1, Q3) & $0.5(0.1,0.7)$ & $0.3(0.1,0.75)$ & 0.91 \\
\hline
\end{tabular}



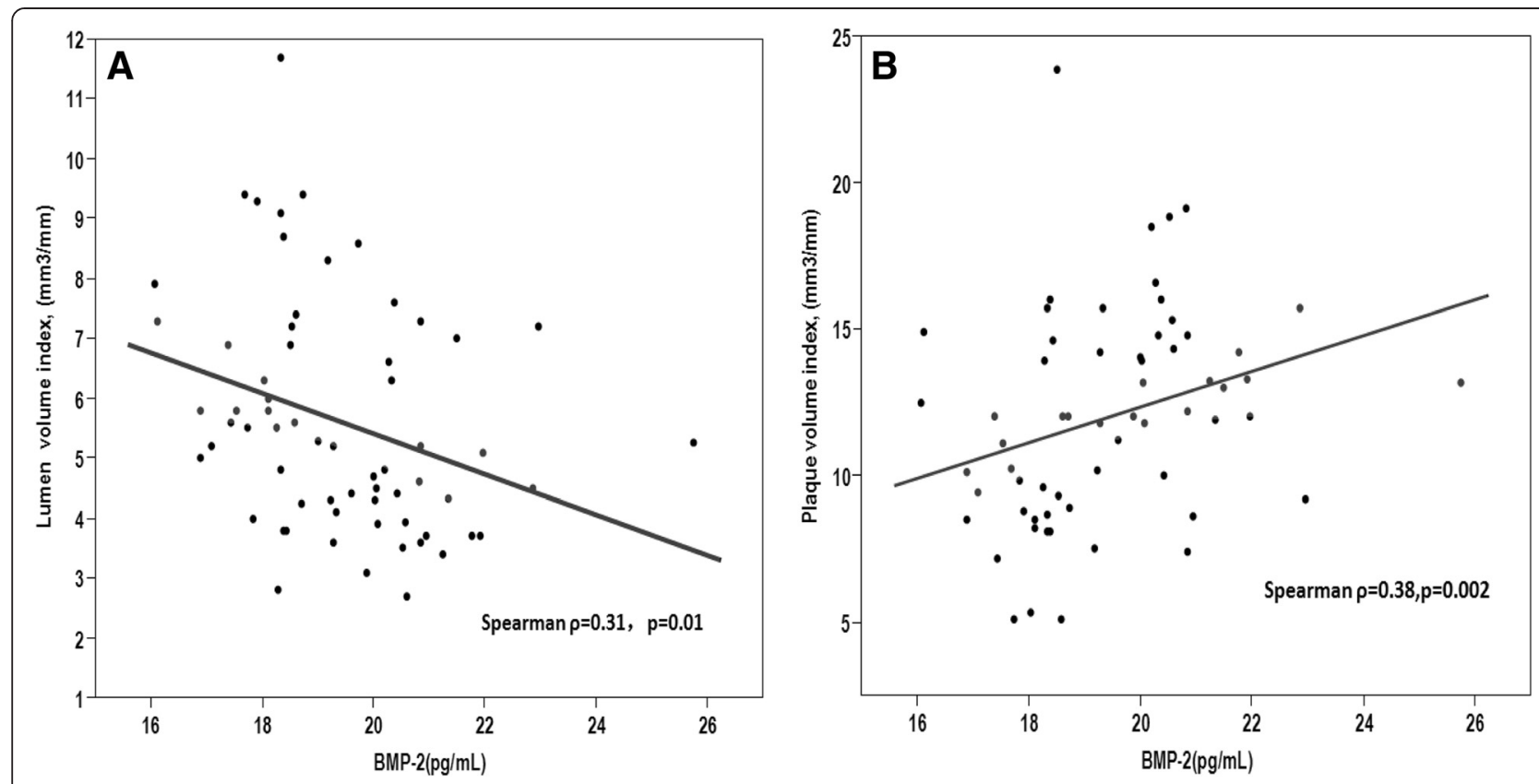

Figure 4 Scatter diagram demonstrating the correlation between BMP-2 levels and lumen volume index, and plaque volume index. (A) Plasma BMP-2 is significantly negatively correlated with lumen volume index. (B) Plasma BMP-2 is significantly positively correlated with plaque volume index.

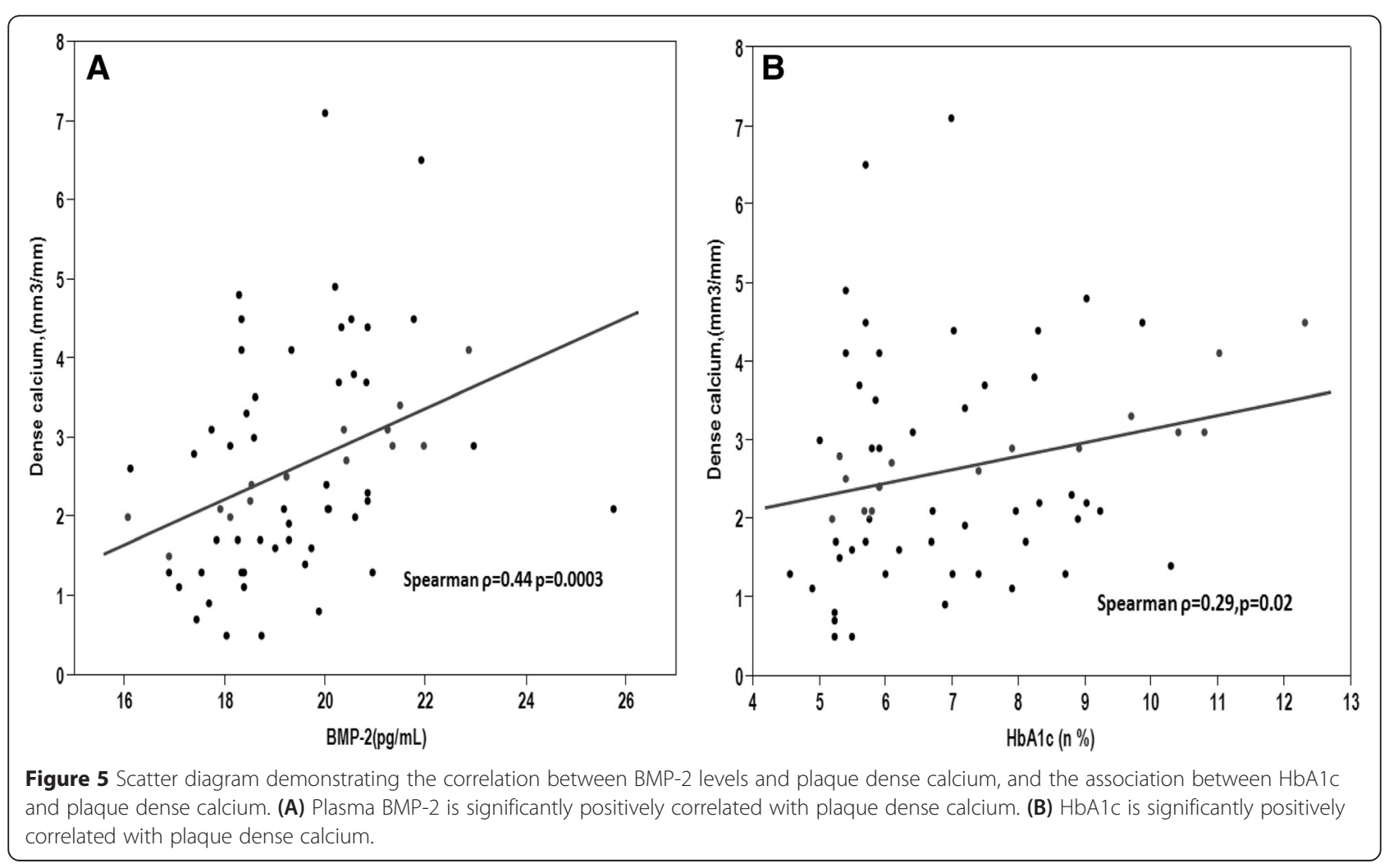


The correlation between BMP- 2 and coronary artery disease Although BMP-2 is important for bone regeneration and is a known mediator of vascular calcification, its role in the regulation of atherosclerotic lesions remains uncertain. BMP-2 exerts proinflammatory, proatherogenic effects and can cause oxidative stress in endothelial cells promoting endothelial cell activation [37]. The current study demonstrates that plasma levels of BMP-2 are significantly higher in patients with CAD. In addition, we showed that BMP-2 levels are positively associated with the severity and extent of coronary stenotic lesions as measured using the SYNTAX score. These results suggest that BMP-2 may play a key role in the pathogenesis of coronary atherosclerosis.

\section{BMP-2 may be involved in hyperglycemia-induced plaque calcification}

Vascular calcification is commonly found in atherosclerosis and is recognized as a marker of plaque burden. The presence of spotty calcification is associated with more extensive and diffuse coronary atherosclerosis and accelerated disease progression despite the use of medical therapy [40]. Arterial stiffness has been shown to be an independent predictor of cardiovascular mortality and an early marker of target organ damage by cardiovascular disease. Vascular calcification is as a major contributor to loss of arterial compliance [41].

Vascular calcification is common in diabetes and is associated with high risk of cardiovascular events. Lesion length, plaque burden, necrotic core, and calcium content have all been shown to be significantly higher in nonculprit lesions of diabetic patients [42]. Though it is known that diabetes is associated with increased vascular calcification, the pathogenesis is not completely understood. VSMCs are thought to drive the calcification process [43]. Elevated glucose levels have been shown to direct osteogenesis by transforming VSMCs and possibly pericytes into osteoblast-like cells [44]. In vitro studies have also shown that high-glucose concentrations are associated with the increased expression of $\mathrm{Cbfa} 1$ and osteocalcin as well as the increased activity of alkaline phosphatase VSMCs. These changes resulted in enhanced calcification and cell proliferation and the increased expression of osteopontin in cultured VSMCs $[22,45]$ as well as in the medial layers of the carotid arteries of streptozotocin-induced diabetic rats [46]. Bostrom and Chen et al. also demonstrated that BMP-2 increased calcification and osteogenic differentiation in calcifying VSMCs, and that exogenous BMP-2 significantly increased the calcification in VSMCs [21,47]. In this study, our IVUS finding showed that plasma BMP-2 levels correlated positively with plaque dense calcium, which itself correlated positively with plasma HbA1c. These findings suggests that BMP-2 may have a role in hyperglycemia-induced calcification, though the precise molecular mechanisms involved require further study.

\section{Limitations}

This study was limited by a small sample size that presented to a University Hospital, likely to constitute a unique cohort, which may restrict the generalizability of our results. The cross-sectional design of the study also makes determining a causal relationship between BMP-2 and $\mathrm{CAD}$ challenging. Further prospective studies are required to determine the role that elevated BMP-2 levels have on clinical outcomes in patients with CAD.

\section{Conclusion}

BMP-2 levels are increased in CAD patients with T2DM and correlate positively with the extent and complexity of coronary atherosclerotic disease as well as the degree of plaque calcification. These finding suggest that BMP-2 may be an important mediator of hyperglycemia-induced plaque progression and calcification.

\section{Competing interests}

The authors declare that they have no competing interests.

\section{Authors' contributions}

MZ conceived and designed the study and wrote the first draft of the paper; JDS and F-LW wrote sections of the manuscript and critically reviewed the paper for intellectual content. MZ, L-XS and XW carried out the experiments and acquired the data; JZ and L-PL performed statistical analyses; J-HL handled funding and supervision. All authors read and approved the final manuscript.

\section{Acknowledgements}

This work was supported by Chinese National Natural and Scientific Funding (No.81441012) and Beijing Natural Science Foundation (No.7132088) and Beijing Novel Star Project on Science and Technology of China (No.2009B38) and Beijing Municipal Training Foundation for Highly-qualified and Technological Talents of Health System (2014-3-038).

\section{Author details}

${ }^{1}$ Department of Cardiology, Beijing Anzhen Hospital, Capital Medical University, Beijing 100029, PR China. ${ }^{2}$ Beijing Institute of Heart, Lung and Blood Vessel Disease, Beijing, China. ${ }^{3}$ Division of Cardiovascular Diseases, Mayo College of Medicine, Rochester, MN, USA. ${ }^{4}$ Department of Nephrology, First Hospital of Tsinghua University, Beijing, China. ${ }^{5}$ Department of Biostatistics, Rutgers School of Public Health, The State University of New Jersey, Piscataway, NJ, USA. ${ }^{6}$ Department of Biostatistics, University at Buffalo, the State University of New York, Buffalo, NY 14214, USA.

Received: 24 February 2015 Accepted: 8 April 2015

Published online: 24 May 2015

\section{References}

1. Sage A, Tintut Y, Garfinkel A, Demer L. Systems biology of vascular calcification. Trends Cardiovasc Med. 2009;19:118-23.

2. Bostrom K, Tsao D, Shen S, Wang Y, Demer LL. Matrix gla protein modulates differentiation induced by bone morphogenetic protein-2 in c3h10t1/2 cells. J Biol Chem. 2001;276:14044-52.

3. Simões Sato AY, Bub GL, Campos AH. BMP-2 and -4 produced by vascular smooth muscle cells from atherosclerotic lesions induce monocyte chemotaxis through direct BMPRII activation. Atherosclerosis. 2014;235:45-55.

4. Hayashi K, Nakamura S, Nishida W, Sobue K. Bone morphogenetic protein induced Msx1 and Msx2 inhibit myocardin-dependent smooth muscle gene transcription. Mol Cell Biol. 2006;26:9456-94570. 
5. Lagna G, Ku MM, Nguyen PH, Neuman NA, Davis BN, Hata A. Control of phenotypic plasticity of smooth muscle cells by bone morphogenetic protein signaling through the myocardin-related transcription factors. J Biol Chem. 2007;282:37244-55.

6. Yao Y, Bennett BJ, Wang X, Rosenfeld ME, Giachelli C, Lusis AJ, et al. Inhibition of bone morphogenetic proteins protects against atherosclerosis and vascular calcification. Circ Res. 2010;107:485-94.

7. Willette RN, Gu JL, Lysko PG, Anderson KM, Minehart H, Yue T. BMP-2 gene expression and effects on human vascular smooth muscle cells. J Vasc Res. 1999:36:120-5.

8. Li X, Yang HY, Giachelli CM. BMP-2 promotes phosphate uptake, phenotypic modulation, and calcification of human vascular smooth muscle cells. Atherosclerosis. 2008;199:271-7.

9. Fukui N, Zhu Y, Maloney WJ, Clohisy J, Sandell LJ. Stimulation of BMP-2 expression by pro-inflammatory cytokines IL-1 and TNF-alpha in normal and osteoarthritic chondrocytes. J Bone Joint Surg Am. 2003;85-A Suppl 3:59-66.

10. Csiszar A, Ahmad M, Smith KE, Labinskyy N, Gao Q, Kaley G, et al. Bone morphogenetic protein-2 induces proinflammatory endothelial phenotype. Am J Pathol. 2006;168:629-38.

11. Su X, Ao L, Shi Y, Johnson TR, Fullerton DA, Meng X. Oxidized low density lipoprotein induces bone morphogenetic protein-2 in coronary artery endothelial cells via Toll-like receptors 2 and 4. J Biol Chem. 2011;286:12213-20.

12. Holman RR, Sourij H, Califf RM. Cardiovascular outcome trials of glucoselowering drugs or strategies in type 2 diabetes. Lancet. 2014;383:2008-17.

13. Seshasai SR, Kaptoge S, Thompson A, Di Angelantonio E, Gao P, Sarwar N, et al. Diabetes mellitus, fasting glucose, and risk of cause-specific death. N Engl J Med. 2011;364:829-41.

14. Paneni F, Beckman JA, Creager MA, Cosentino F. Diabetes and vascular disease: pathophysiology, clinical consequences, and medical therapy: part I. Eur Heart J. 2013;34:2436-43.

15. Howangyin KY, Silvestre JS. Diabetes mellitus and ischemic diseases: molecular mechanisms of vascular repair dysfunction. Arterioscler Thromb Vasc Biol. 2014;34:1126-35.

16. Abedin $M$, Tintut $Y$, Demer LL. Vascular calcification: mechanisms and clinical ramifications. Arterioscler Thromb Vasc Biol. 2004;24:1161-70.

17. Parhami F, Boström K, Watson K, Demer LL. Role of molecular regulation in vascular calcification. J Atheroscler Thromb. 1996;3:90-4.

18. Shemesh J, Tenenbaum A, Fisman EZ, Koren-Morag N, Grossman E. Coronary calcium in patients with and without diabetes: first manifestation of acute or chronic coronary events is characterized by different calcification patterns. Cardiovasc Diabetol. 2013;12:161.

19. Hruska KA, Mathew S, Saab G. Bone morphogenetic proteins in vascular calcification. Circ Res. 2005:97:105-14.

20. Snell-Bergeon JK, Budoff MJ, Hokanson JE. Vascular calcification in diabetes: mechanisms and implications. Curr Diab Rep. 2013;13:391-402.

21. Boström Kl, Jumabay M, Matveyenko A, Nicholas SB, Yao Y. Activation of vascular bone morphogenetic protein signaling in diabetes mellitus. Circ Res. 2011;108:446-57.

22. Chen NX, Duan D, O'Neill KD, Moe SM. High glucose increases the expression of Cbfa1 and BMP-2 and enhances the calcification of vascular smooth muscle cells. Nephrol Dial Transplant. 2006;21:3435-42.

23. Zhang M, Zhou SH, Zhao S, Li XP, Liu LP, Shen XQ. Pioglitazone can downregulate bone morphogenetic protein-2 expression induced by high glucose in human umbilical vein endothelial cells. Pharmacology. 2008:81:312-6

24. Alberti KG, Zimmet PZ. Definition, diagnosis and classification of diabetes mellitus and its complications. Part 1: diagnosis and classification of diabetes mellitus provisional report of a WHO consultation. Diabet Med. 1998;15:539-53.

25. Libby $P$, Theroux P. Pathophysiology of coronary artery disease. Circulation. 2005;111:3481-8.

26. Fisher LD, Kennedy JW, Chaitman BR, Ryan TJ, McCabe C, Weiner D, et al Diagnostic quantification of CASS (Coronary Artery Surgery Study) clinical and exercise test results in determining presence and extent of coronary artery disease. A multivariate approach. Circulation. 1981;63:987-1000.

27. Coronary Artery Surgery Study (CASS). A randomized trial of coronary artery bypass surgery. Survival data. Circulation. 1983;68:939-50.

28. Head SJ, Farooq V, Serruys PW, Kappetein AP. The SYNTAX score and its clinical implications. Heart. 2014;100:169-77.

29. Garcia-Garcia HM, Mintz GS, Lerman A, Vince DG, Margolis MP, van Es GA, et al. Tissue characterisation using intravascular radiofrequency data analysis: recommendations for acquisition, analysis, interpretation and reporting. Eurolntervention. 2009;5:177-89.

30. Nasu K, Tsuchikane E, Katoh O, Vince DG, Virmani R, Surmely JF, et al. Accuracy of in vivo coronary plaque morphology assessment: a validation study of in vivo virtual histology compared with in vitro histopathology. J Am Coll Cardiol. 2006;47:2405-12.

31. Matsuo Y, Cassar A, Li J, Flammer AJ, Choi BJ, Herrmann J, et al. Repeated episodes of thrombosis as a potential mechanism of plaque progression in cardiac allograft vasculopathy. Eur Heart J. 2013;34:2905-15.

32. Zhang $\mathrm{H}$, Bradley A. Mice deficient for BMP2 are nonviable and have defects in amnion/chorion and cardiac development. Development. 1996;122:2977-86.

33. Csiszar A, Smith KE, Koller A, Kaley G, Edwards JG, Ungvari Z. Regulation of bone morphogenetic protein-2 expression in endothelial cells: role of nuclear factor-kappaB activation by tumor necrosis factor-alpha, $\mathrm{H} 2 \mathrm{O} 2$, and high intravascular pressure. Circulation. 2005;111:2364-72.

34. Shanahan CM, Cary NR, Metcalfe JC, Weissberg PL. High expression of genes for calcification-regulating proteins in human atherosclerotic plaques. J Clin Invest. 1994:93:2393-402.

35. Dhore CR, Cleutjens JP, Lutgens E, Cleutjens KB, Geusens PP, Kitslaar PJ, et al. Differential expression of bone matrix regulatory proteins in human atherosclerotic plaques. Arterioscler Thromb Arterioscler Thromb Vasc Biol. 2001;21:1998-2003.

36. Nakagawa Y, Ikeda K, Akakabe Y, Koide M, Uraoka M, Yutaka KT, et al. Paracrine osteogenic signals via bone morphogenetic protein-2 accelerate the atherosclerotic intimal calcification in vivo. Arterioscler Thromb Vasc Biol. 2010;30:1908-15

37. Zhang M, Yang M, Liu LP, Lau WB, Gao H, Xin MK, et al. BMP-2 overexpression augments vascular smooth muscle cell motility by upregulating myosin Va via Erk signaling. Oxid Med Cell Longev. 2014;2014:294150.

38. Asaba K, Iwasaki Y, Asai M, Yoshida M, Nigawara T, Kambayashi M, et al. High glucose activates pituitary proopiomelanocortin gene expression: possible role of free radical-sensitive transcription factors. Diabetes Metab Res Rev. 2007;23:317-23.

39. Yamagishi S, Nakamura K, Matsui T, Takenaka K, Jinnouchi Y, Imaizumi T. Cardiovascular disease in diabetes. Mini Rev Med Chem. 2006;6:313-8.

40. Kataoka Y, Wolski K, Uno K, Puri R, Tuzcu EM, Nissen SE, et al. Spotty calcification as a marker of accelerated progression of coronary atherosclerosis: insights from serial intravascular ultrasound. J Am Coll Cardiol. 2012;59:1592-7.

41. Sung KC, Lim YH, Park S, Kang SM, Park JB, Kim BJ, et al. Arterial stiffness, fatty liver and the presence of coronary artery calcium in a large population cohort. Cardiovasc Diabetol. 2013;12:162.

42. Marso SP, Mercado N, Maehara A, Weisz G, Mintz GS, McPherson J, et al. Plaque composition and clinical outcomes in acute coronary syndrome patients with metabolic syndrome or diabetes. JACC Cardiovasc Imaging 2012;5:S42-52.

43. Liu Y, Shanahan CM. Signalling pathways and vascular calcification. Front Biosci. 2011;16:1302-14.

44. Hayden MR, Tyagi SC, Kolb L, Sowers JR, Khanna R. Vascular ossificationcalcification in metabolic syndrome, type 2 diabetes mellitus, chronic kidney disease, and calciphylaxis-calcific uremic arteriolopathy: the emerging role of sodium thiosulfate. Cardiovasc Diabetol. 2005;18(4):4.

45. Sodhi CP, Phadke SA, Batlle D, Sahai A. Hypoxia stimulates osteopontin expression and proliferation of cultured vascular smooth muscle cells: potentiation by high glucose. Diabetes. 2001;50:1482-90.

46. Mori S, Takemoto M, Yokote K, Asaumi S, Saito Y. Hyperglycaemia-induced alteration of vascular smooth muscle phenotype. J Diabetes Complications. 2002;16:65-8.

47. Bostrom K, Watson KE, Horn S, Wortham C, Herman IM, Demer LL. Bone morphogenetic protein expression in human atherosclerotic lesions. J Clin Invest. 1993;91:1800-9. 